Tre statement of Sir James Swinburne ${ }^{1}$ that the heat engine cycles are concerned, because there are diagrams which give a very convenient representation

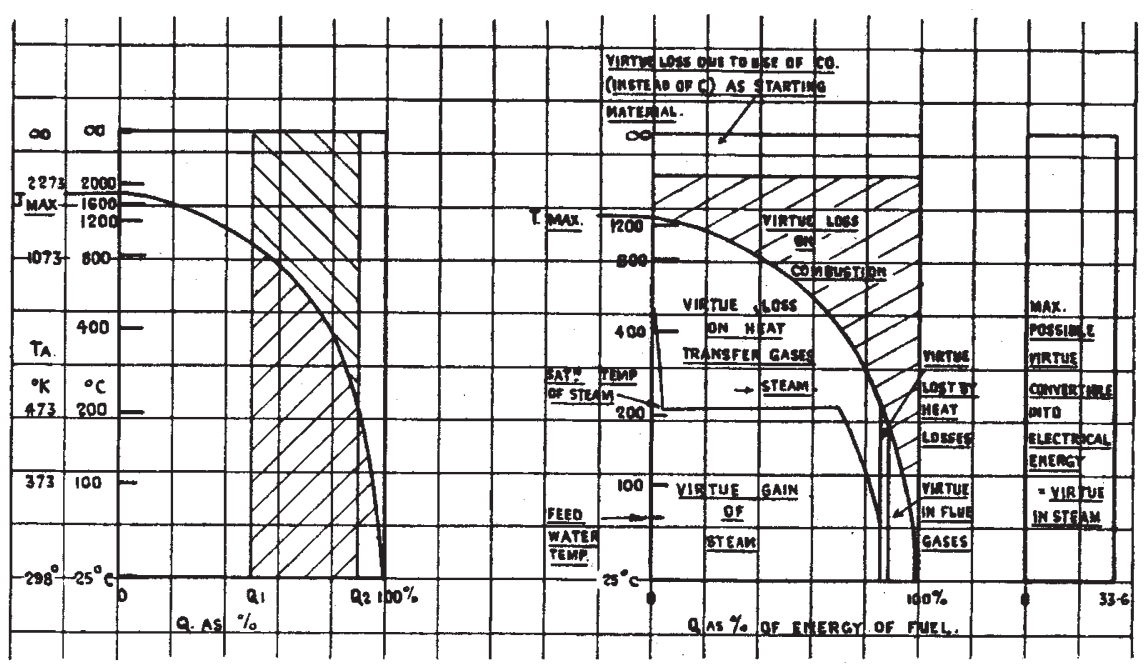

Fig. 1.

Fig. 2. VIRTde DIAGRAM FOR INDUSTRIAL BOLLER AND IDEAL HEAT ENGINE.

\section{The Nature of Entropy} engineer understands entropy is correct so far as

and that for the mechanical or electrical energy, which are lines coinciding with $T_{A}=\infty$.

When considering a chemical reaction such as combustion, it is necessary to take into account the fact that there is an entropy change between the initial and final compounds when both are at room temperature. If this is done by representing the initial virtue of various fuels by lines differing slightly from $T_{A}=\infty$ (the correction is of importance only in the case of carbon monoxide and hydrogen) then the irreversibility of adiabatic combustion is the area between this initial virtue and the curve for the 'theoretical' hot products of combustion.

It thus becomes possible to represent on one diagram the irreversibility of the successive stages of (1) combustion of a fuel, (2) heat transfer from the hot gases to steam, and (3) conversion of the steam energy into mechanical work. An industrial exam. ple is shown in Fig. 2.

These ideas have considerable practical importance in these times when energy conservation is so vital to the war effort.

conversion processes in engineering in which Second Law of Thermodynamics is of equal importance and for which no comparable diagrams exist, heat transfer and combustion reactions being typical examples.

It is possible to construct simple diagrams relating to these processes which bring out very clearly the practical significance of entropy and of its negative, for which the term 'virtue' is suggested. The abscissa is $Q$, the 'available' heat of the working substance at a given temperature $T_{\boldsymbol{A}}\left({ }^{\circ} \mathrm{K}\right.$.) expressed in any convenient units. The ordinate is $1 / T_{A}$. This is conveniently plotted downwards so that the line $T_{A}=\infty$ is at the top of the diagram and the line $T_{A}=298^{\circ} \mathrm{K}$. at the bottom. Suppose now that we have a body (for example, of combustion gas) of constant specific heat at temperature $T_{\max }$. The graph of the available heat is then a rectangular hyperbola crossing the lowest abscissa at the point representing its total heat content above room temperature and crossing the line $Q=0$ at the point corresponding to $T_{\max }$. (see Fig. 1). The area between the curve, the lines $Q=Q_{1}$ and $Q=Q_{2}$ and the line $T_{A}=\infty$ is the entropy change of the body on losing heat $Q_{2}-Q_{1}$. It is, however, much easier to regard the area between the curve, the line $T_{A}=298^{\circ} \mathrm{K}$. and $Q=Q_{1} Q=Q_{2}$ as the 'virtue' of this amount of heat, since we can then express the Second Law of Thermodynamics by the statement that the virtue of a certain quantity of heat must always decrease in any physical process.

It is at once possible to represent heat transfer by drawing the curves for the body losing and the body receiving heat with corresponding values of $Q$. The area between the curves is then the irreversibility of the process or the loss of virtue of the heat.

Heat engine processes may also be conveniently represented, since the condition for the perfect engine that entropy does not increase is represented by an equal area under the curve for the working fluid,

\section{British Coal Utilisation \\ Research Association, \\ Experimental Station, Rickett Street, London, S.W.6.}

${ }^{1}$ NATURE, 151, 335 (1943).

\section{A Diatom New to Britain}

IN a previous communication ${ }^{1}$ one of us described of Cheshire and Flintshire about two miles south of Malpas. The diatom flora of this same pit has recently been the subject of an investigation by us. Preliminary observations indicate that the majority of the diatoms occurring in the pit are exceedingly minute and that their frustules are poorly silicified. Generally speaking, the assemblage of forms is of the type to be expected in a brackish habitat. A complete account of the diatoms found must await later publication. One diatom, however, which we have identified as Navicula incerta Grun. (see accompanying illustration), is of special interest and deserves notice now because it is a purely marine form and so far as can be ascertained from the sources of information at our disposal ${ }^{3,4,5,6}$ has not previously been recorded from British waters.

Navicula incerta was found in a gather. ing collected from the brine-pit on June 21 , ( $\times 1450)$. 1942. An analysis of the pit water at tion : 9,500 parts of sodium chloride per 100,000 ; 10,400 parts of dissolved solids per 100,000, and a $p H$ value of $7 \cdot 5$ (Lovibond comparator). the insect fauna of a brine-pit ${ }^{2}$ situated on the border that time showed the following composi- 